\title{
VERBANDSGELDBUBEN IM FINANZSEKTOR: EINE KUMULATION ZWEIFELHAFTER OBERGRENZEN
}

\author{
Pecuniary sanctions against corporations in the financial sector: \\ a compounding of dubious upper limits
}

\author{
SUZAN DENISE HÜTTEMANN*
}

\begin{abstract}
This contribution covers the methods by which the maximum amount of pecuniary sanctions against corporations in the financial sector is determined. The alternative recourse to absolute, turnover-based and profit-based upper limits combines problematic regulatory developments of recent times. This contribution advocates for a systematic approach that differentiates strictly between the confiscatory and the sanctioning part; the upper limit of the sanctioning part should be determined by the abstract gravity of the violation.
\end{abstract}

Keywords: Financial sector, corporate sanctions, German Securities Trading Act, multiplicative pecuniary sanctions, guilt principle

\section{EINLEITUNG}

Der folgende Beitrag befasst sich mit Verbandsgeldbußen im Finanzsektor. Im Zentrum wird dabei die Methode der Obergrenzenbestimmung stehen, die zahlreiche verfassungsrechtliche, europarechtliche, aber auch systematische Fragen aufwirft. Diese werden anhand der Regelungen des Wertpapierhandelsgesetzes illustriert, nur gelegentlich sollen auch andere Gesetze wie etwa das Kreditwesengesetz oder das Sanierungs- und Abwicklungsgesetz berücksichtigt werden.

Das WpHG sanktioniert verschiedenste Verstöße in den $\S \S 120,127$ und 130 als Ordnungswidrigkeiten. Insgesamt handelt es sich um ein sehr komplexes Regelungsgefüge mit etwa 400 verschiedenen Bußgeldtatbeständen. ${ }^{1}$ Daneben finden sich zahlreiche Straftatbestände in $\S 119 \mathrm{WpHG}$, die ebenfalls Anknüpfungstat einer Verbandsgeldbuße darstellen können. ${ }^{2}$ Die folgende Darstellung soll sich bewusst auf

* Die Autorin ist Inhaberin der Juniorprofessur für Strafrecht an der Universität Mannheim.

1 Vgl. SzESNY, André M. - KuTHE, Thorsten (Hrsg.): Kapitalmarkt Compliance. Heidelberg, 2. Aufl., 2018, 30. Kapitel, Rn. 39.

2 Die Obergrenzen der Ordungswidrigkeitentatbestände gelten für diese nicht direkt. Umstr. ist allerdings, welche Obergrenzen für diese gelten sollen bzw. wie sich diese bei Zusammentreffen mit Ordnungswidrigkeiten nach $\S 17$ Abs. 2 OWiG bestimmen sollen, vgl. dazu ACHENBACH, Hans: Neue Sanktionen im Finanzmarktrecht - alte und neue Zweifelsfragen. In: wistra, 2018, 13, 21 einerseits, ders.: Das Höchstmaß der Verbandsgeldbuße wegen Kapitalmarkt-Straftaten. In: WM, 2018, 1337, 1339 f. andererseits. 
einige wenige, besonders interessant erscheinende, Aspekte systematischer Natur beschränken.

In den vergangenen Jahren kam es im Finanzmarktrecht zu einer grundlegenden Umgestaltung des Ordnungswidrigkeitenregimes, welche zum Teil, aber nicht vollständig, europarechtlich determiniert war. Die wesentlichen Neuerungen wurden im Zuge des Ersten und Zweiten Finanzmarktregulierungsgesetzes von 2016 bzw. 2017 eingeführt. Erste Änderungen vergleichbarer Art fanden sich aber bereits 2015 mit dem Gesetz zur Umsetzung der Transparenzrichtlinie-Änderungsrichtlinie. ${ }^{3}$ Die im Zuge dessen eingeführten Neuerungen spiegeln dabei nicht etwa nur einen Trend im Bereich der Finanzmarktregulierung wider, sondern sind bezeichnend für eine generelle Tendenz, die sich auch in vergleichbaren Regelungen in so unterschiedlichen Rechtsbereichen wie dem Datenschutzrecht ${ }^{4}$, dem Energiewirtschaftsrecht ${ }^{5}$ oder auch dem Geldwäschegesetz ${ }^{6}$ niederschlägt. Besondere systematische Probleme bereiten hierbei die Parameter für die Bestimmung der Bußgeldrahmenobergrenze sowie die Verortung der abschöpfenden Funktion von Geldbußen. Bei der Verletzung von Offenlegungspflichten kapitalmarktorientierter Kapitalgesellschaften finden sich vergleichbare Regelungsmechanismen über den Bereich des Ordnungswidrigkeitenrechts hinaus ${ }^{7}$ auch im Bereich der Ordnungsmittel, nämlich bei Ordnungsgeldverfahren bei Verletzung von Offenlegungspflichten kapitalmarktorientierter Kapitalgesellschaften. ${ }^{8}$

\section{Geldbuben gegen natürliche Personen nach deM WPHG}

Um die Problematik zu erörtern, soll zunächst der Prototyp einer Geldbuße nach dem WpHG dargestellt werden: Für natürliche Personen findet sich für die verschiedenen Arten von Ordnungswidrigkeiten in $\S 120$ Abs. 17-22a WpHG regelmäßig eine betragsmäßige Obergrenze. Diese soll nach $§ 120$ Abs. 25 WpHG in Abweichung von

3 Vgl. die durch Art. 1 Nr. 30 lit. c des Gesetzes zur Umsetzung der TransparenzrichtlinieÄnderungsrichtlinie vorgenommene Änderung des $\S 39 \mathrm{WpHG}$ a.F.

4 Vgl. Art. 83 Abs. 4-6 DSGVO.

5 Vgl. § 95 Abs. 2 EnWG, dessen mehrerlösorientierte Obergrenze bereits 2005 eingeführt wurde und nicht europarechtlich determiniert ist, sondern eine Orientierung an $\S 81 \mathrm{Abs}$. 2 GWB darstellte, vgl. BT-Drs. 15/3917, S. 74.

6 Vgl. § 56 Abs. 3 S. 1 Nr. 2 GwG.

7 Vgl. § 334 Abs. 3 S. 1 Nr. 2 HGB.

8 Vgl. § 335 Abs. 1a HGB, welcher der Umsetzung von Art. 28b und 28c der Transparenzrichtlinie in der Fassung der Richtlinie 2013/50/EU dienen soll, vgl. BT-Drs. 58/5010, S. 57. Nach dem Bundesverfassungsgericht ist der strenge Maßstab des Art. 103 Abs. 2 GG auch auf das Ordnungsgeld anzuwenden, da es auch eine repressive Sanktionswirkung habe, vgl. BVerfG DStR 2014, 540, 541; die Höchstmaßbestimmung war in der Entscheidung jedoch nicht von Bedeutung; vgl zur Wirkung auch DANNECKER, Gerhard - KERN, Ursula: In: STAUB, Hermann (Begr.): Handelsgesetzbuch. Bd. 7/2, 5. Aufl., Berlin, 2012, $\S 335 \mathrm{Rn} .12$. 
den allgemeinen Regeln ( $§ 17$ Abs. $2 \mathrm{OWiG}$ ) bei den meisten Verstößen für vorsätzliche und fahrlässige Begehung gleichermaßen gelten. ${ }^{9}$ Zudem findet sich darüber hinaus regelmäßig, wenn auch nicht immer, eine alternative Höchstmaßbestimmung, nämliche eine relative Obergrenze, bei welcher der aus dem Verstoß gezogene wirtschaftliche Vorteil mit einem bestimmten Faktor multipliziert wird. Maßgeblich ist dann als Obergrenze das jeweils höhere Maß.

\section{VERBANDSGELDBUBEN NACH DEM WPHG}

\subsection{Betragsmäßige Obergrenzen}

Für juristische Personen und Personenvereinigungen kann eine Verbandsgeldbuße nach $\S 30 \mathrm{OWiG}$ in Anknüpfung an eine entsprechende Ordnungswidrigkeit einer Leitungsperson festgesetzt werden. ${ }^{10}$ Hierfür hat der Gesetzgeber in $\S 120$ Abs. 17-22a WpHG nicht nur zwei, sondern gleich drei alternative Obergrenzen vorgesehen, wobei ebenfalls jeweils der höchste Betrag maßgeblich ist. Zunächst findet sich auch für Verbände eine absolute, also betragsmäßige, Obergrenze. Diese liegt in seltenen Fällen bei der gleichen Höhe wie für natürliche Personen, zumeist aber bei einem Vielfachen, teilweise sogar beim bis zum 6,67-fachen (etwa in $\S 120$ Abs. 19, 22a WpHG). Die höchste betragsmäßige Obergrenze für juristische Personen und Personenvereinigungen im WpHG liegt bei 15 Millionen Euro (vgl. § 120 Abs. 18 S. 2 Nr. 1, Abs. $21 \mathrm{~S}$. 2 Nr. 2 WpHG), im KWG sogar bei 20 Millionen Euro (§ 56 Abs. 6a S. 2 Nr. 2 KWG).

\subsection{Umsatzbezogene Obergrenzen}

Alternativ kann bei Verbandsgeldbußen die Obergrenze aus dem Umsatz abgeleitet werden (sog. Bruchteilsgeldbuße). Im Finanzmarktrecht wird meist auf den Gesamtumsatz des der Behördenentscheidung vorausgehenden Geschäftsjahrs abgestellt. Die Obergrenze beträgt im WpHG zwischen $1,5^{11}$ und $15^{12}$ Prozent. Der Umsatz ist dabei für verschiedene Sektoren (Kreditinstitute u.ä., Versicherungsunternehmen und sonstige Unternehmen) je nach Rechnungslegungsvorschriften in $\S 120$ Abs. $23 \mathrm{WpHG}$ unterschiedlich definiert. ${ }^{13}$ Eine vergleichbare umsatzbezogene Obergrenze bereitet im Kartellrecht ( $\$ 81 \mathrm{c}$ Abs. 2, 4 und 5 GWB bzw. zuvor $\S 81$ Abs. 4 GWB a.F.) erhebliche Probleme. Allgemein wird eine umsatzbezogene Obergrenze unter anderem kritisiert, weil sie - ähnlich wie der wandernde Strafrahmen bei der für verfassungswidrig erklärten Vermögensstrafe ${ }^{14}-$ nicht im Vorhinein

9 Vgl. auch BÖSE, Martin - JANSEN, Scarlett: In: SCHWARK, Eberhard - ZIMMER, Daniel (Hrsg.): Kapitalmarktrechtskommentar, 5. Aufl., München, 2020, WpHG § 120 Rn. 98.

10 Vgl. zu diesbezüglichen Fragen, insbesondere zur Europarechtskonformität SPOERR, Wolfgang: In: AssmanN, Heinz Dieter - SCHNEIDER, Uwe H. - MÜLBERT, Peter O. (Hrsg.): Wertpapierhandelsrecht, 7. Aufl., Köln, 2019, WpHG § 120 Rn. 403 ff.

11 In $\S 120$ Abs. 22a WpHG i.V.m. $\S 17$ Abs. 2 OWiG.

12 In $\S 120$ Abs. 18 S. 2 Nr. 1 WpHG.

13 Vgl. BT-Drs. 18/5010, S. 53.

14 BVerfGE 105, 35. 
bestimmt sei und dem Rechtsanwender ihm nicht zukommende Funktionen zuweise. Dadurch soll der Bestimmtheitsgrundsatz aus Art. 103 Abs. 2 GG, der auch für Ordnungswidrigkeiten gilt, ${ }^{15}$ verletzt sein. ${ }^{16}$ Eine absolute - vom Bundesgerichtshof „starr“ genannte - Obergrenze soll der Bestimmtheitsgrundsatz nach der Rechtsprechung nicht zwingend erfordern; es genüge eine relative - vom Bundesgerichtshof als „fest" bezeichnete - Obergrenze. Auf die Bestimmtheitsproblematik werde ich noch bei der vorteilsbezogenen Obergrenze eingehen. An dieser Stelle sei nur gesagt, dass ich bei der umsatzbezogenen Obergrenze Bedenken im Hinblick auf den Bestimmtheitsgrundsatz nur im Konkreten, nicht aber im Grundsätzlichen teile.

Die deutsche Rechtsprechung im Kartellrecht sieht keine Verletzung des Bestimmtheitsgrundsatzes, soweit der Höchstbetrag als Obergrenze und nicht wie im Europarecht als Kappungsgrenze ${ }^{17}$ behandelt wird. ${ }^{18}$

Zudem sind im vollständig europarechtlich determinierten Bereich - zu welchem die Bußgeldvorschriften des WpHG teilweise zählen ${ }^{19}$ - wegen des grundsätzlichen Anwendungsvorrangs des Europarechts nur der europäische Gesetzlichkeitsgrundsat $^{20}$ sowie der integrationsfeste Kern des nationalen Bestimmtheitsgrundsatzes ${ }^{21}$

15 Vgl. BVerfG NJW 1975, 727 (730); DANNECKER, Gerhard - SCHUHR, Jan C.: In: CiRENER, Gabriele - RADTKE, Henning - RISSING-VAN SAAN, Ruth - RÖNNAU, Thomas SCHLUCKEBIER, Wilhelm (Hrsg.): Strafgesetzbuch - Leipziger Kommentar. Großkommentar. Bd. 1, 13. Aufl., Berlin, 2020, § 1, Rn. 65, 69 ff.; BÜLTE, Jens: Ordnungswidrigkeitenrecht. 6. Aufl., 2020, $§ 2$ Rn. 2 ff.; MITSCH, Wolfgang: In: KK-OWiG. 5. Aufl., 2018, Einl. Rn. 121 ff.; zur Geltung für Rechtsfolgen vgl. DANNECKER, Gerhard: Nullum crimen, nulla poena sine lege und seine Geltung im Allgemeinen Teil des Strafrechts. In: DANNECKER, Gerhard (Hrsg.): Festschrift für Otto. Köln, 2007, 25 (36 ff.).

16 ACHENBACH: wistra, 2018, 13, 16; krit. bezüglich Bruchteilsgeldbußen, allerdings ohne eine Verletzung des Bestimmtheitsgrundsatzes zu behaupten, auch BMJV, Handbuch des Nebenstrafrechts. 3. Aufl., 2018, Rn. 234.

17 Vgl. krit. BIERMANN, Jörg: In: IMMENGA, Ulrich - MESTMÄCKER, Ernst-Joachim (Hrsg.): Wettbewerbsrecht. Band 1, 6. Aufl., München, 2019, Art. 23 VO (EG) Nr. 1/2003 Rn. 115; KALlMAYER, Axel - HAUPT, Heiko: Die Urteile des EuG zum Fernwärmerohrkartell - Die Bußgeldleitlinien der Kommission auf dem Prüfstand. EuZW, 2002, 677, 681 f.

18 Vgl. BGH NZKart 2013, 195, 197 ff. - Grauzementkartell; BIERMANN, a.a.O., GWB § 81, Rn. 406 ff.; a.A.: Vollmer, Christof: In: MonTAG, Frank - SÄCKER, Franz Jürgen BIEN, Florian - MEIER-BECK, Peter (Hrsg.): Münchener Kommentar zum Wettbewerbsrecht. 3. Aufl., München, 2020, GWB § 81 Rn. 137 ff.; BARTH, Christoph - BudDE, Stephanie: „Die Strafe soll nicht größer sein als die Schuld“ Zum Urteil des BGH in Sachen Grauzement und den neuen Leitlinien für die Bußgeldzumessung. NZKart, 2013, 311, 312 f.; krit. wohl auch RÖNNAU, Thomas/WEGNER, Kilian: In: MEYER, Andreas - VEIL, Thomas - RÖNNAU, Rüdiger (Hrsg.): Handbuch zum Marktmissbrauchsrecht. München, 2018, § 29 Rn. 38.

19 Offenlassend ACHENBACH: wistra, 2018, 13, 16.

20 Vgl. zur möglichen Überprüfung der GRC durch das Bundesverfassungsgericht BVerfG EuZW 2019, 1035 (Recht auf Vergessen II).

21 Zum Prüfmaßstab des integrationsfesten Kerns der Grundrechte grundlegend BVerfG NJW, 2016, 1149, 1150 ff. - Solange III; vgl. auch BVerfG NJOZ, 2016, 1879; der EuGH 
anwendbar. Nur bei etwaigen Spielräumen wären diese vom Gesetzgeber nach den Vorgaben des Grundgesetzes auszugestalten. ${ }^{22}$ Der Europäische Gerichtshof folgt jedoch einem gänzlich anderen Verständnis des Bestimmtheitsgebots, welches zunächst aus den gemeinsamen Verfassungsüberlieferungen und Art. 7 Abs. 1 EMRK hergeleitet werden konnte und mittlerweile auch in Art. 49 Abs. 1 GRC verankert ist. ${ }^{23} \mathrm{We}-$ sentliche Funktion des Gesetzlichkeitsprinzips ist danach die Rechtssicherheit, die eine hinreichende Vorhersehbarkeit von Tat und möglicher Sanktion erfordert. Bei der Sanktion soll jedoch keine exakte Determinierung erforderlich sein, sondern eine Bestimmbarkeit ausreichen. Diese gilt trotz eines weiten Ermessens als gewahrt, wenn die Praxis die maßgebenden Kriterien hinreichend konkretisiert hat. In der praktischen Konsequenz wird hierdurch etwa die Heranziehung von bloßen Kappungsgrenzen möglich, sodass Art. 23 Abs. 2 VO(EG) 1/2003 als solche verstanden wird. ${ }^{24}$ Der Bußgeldrahmen ist daher für die Kommission insoweit unbegrenzt; nur der Endbetrag wird gekappt. In der theoretischen Konzeption bedeutet dies einen weitgehenden Verzicht auf die Funktionsbegrenzung von Judikative und Exekutive durch den Bestimmtheitsgrundsatz. Dass die Legislative die wesentlichen Voraussetzungen der Strafbarkeit in hinreichender Regelungsdichte festlegen muss, ${ }^{25}$ wird nicht im gleichen Maße als Ausprägung des europarechtlichen Bestimmtheitsgrundsatzes verstanden. ${ }^{26}$

Die umsatzbezogene Obergrenze im WpHG enthält aber im Detail eine gegenüber anderen Bereichen, insbesondere auch dem Kartellrecht, abweichende Regelung über die Maßgeblichkeit des Konzernumsatzes in bestimmten Fällen. Handelt es sich bei der juristischen Person um ein Mutterunternehmen oder um eine Tochtergesellschaft, so ist grundsätzlich anstelle des Gesamtumsatzes der juristischen Person selbst der jeweilige Gesamtbetrag in dem Konzernabschluss des Mutterunternehmens maßgeblich, der für den größten Kreis von Unternehmen aufgestellt wird. Hierin sind zwei Konstellationen genannt: die Bebußung des Mutterunternehmens und die Bebußung des Tochterunternehmens in Anknüpfung an eine Tat ihrer jeweils eigenen Leitungsperson. Besonders problematisch ist die Heranziehung des Konzernumsatzes zur Verhängung einer Geldbuße gegen die Tochtergesellschaft. ${ }^{27}$ Denn die Rechtsträger der Unternehmen in einem Konzern sind grundsätzlich unabhängig; auch gibt es keine faktische oder rechtliche Vermutung dafür, dass die Muttergesellschaft bestimmenden Einfluss hätte. Schon im Kartellbußgeldrecht ist das Abstellen auf den Konzernumsatz

scheint dies im Ansatz zu akzeptieren, vgl. EuGH (GK), 5. 12. 2017, Rs. - C-42/17, ECLI:EU:C:2017:936, Rn. 46 f., 61 (M.A.S. und M.B.; Satzger), EuCLR, 2018, 317, 330.

22 Vgl. etwa BVerfG EuZW, 2019, 1021 (Recht auf Vergessen I).

23 Vgl. EuGH, 22. 05. 2008, Rs. C-266/06 P, Slg. 1008, I-00081, Rn. 44 ff. (Evonik Degussa $\mathrm{GmbH}$ ).

24 EuG, 20. 03. 2002, Rs. T-23/99, Slg. 2002, II-01705 Rn. 286 ff. (LR AF 1998); EuG, 20. 03. 2002, Rs. T-9/99, Slg. 2002, II-01487 Rn. 450 ff. (HFB Holding u.a.).

25 Dazu aus deutschrechtlicher Sicht REMMERT, Barbara: In: MAUNZ, Theodor - DÜRIG, Günter (Begr.): Grundgesetz. Loseblatt, Stand: 92. EL 2020, Art. 103 Abs. 2 Rn. 101 ff.

26 Vgl. krit. VOET VAN VORMIZEELE, Philipp: Kartellrecht und Verfassungsrecht. NZKart, 2013, 386, 391 m.w.N. auch aus der Rspr.

27 Vgl. ACHENBACH: wistra, 2018, 13, $17 \mathrm{f}$. 
problematisch. ${ }^{28}$ Allerdings sind dort zumindest bestimmte eingrenzende materielle Kriterien erforderlich. § 81c Abs. 5 S. 1 GWB fordert ausdrücklich das Operieren als wirtschaftliche Einheit, das sich an einem bestimmenden Einfluss auf die Tochtergesellschaft ablesen lässt. ${ }^{29}$ Das europäische System, insbesondere die europäische Rechtsprechung, folgte schon zuvor dem Konzept der ,wirtschaftlichen Einheit“. Anforderungen diesbezüglich fehlen im deutschen Bußgeldrecht im Finanzmarktbereich gänzlich. ${ }^{30}$ Auch würden entsprechende Kriterien die Problematik kaum entschärfen. Denn der bestimmende Einfluss macht die Tochtergesellschaft nicht wirtschaftlich leistungsfähiger und vergrößert auch nicht das Unrecht der Tat. Die Muttergesellschaft wird so jedoch faktisch gezwungen, auf das Handeln der Tochter Einfluss zu nehmen und mittelbar verantwortlich einzustehen. Immerhin ist im WpHG die unmittelbare (alleinige oder gesamtschuldnerische) Bebußung des Mutterunternehmens für einen Verstoß einer Leitungsperson der Tochtergesellschaft nicht möglich, anders als bei Geldbußen der Kommission im Kartellrecht. Der Entwurf für ein Verbandssanktionengesetz, ${ }^{31}$ der derzeit in Deutschland die rechtspolitische Diskussion bestimmt, stellt dem Kartellrecht vergleichbare Kriterien auf (nämlich das Vorliegen einer wirtschaftlichen Einheit und des bestimmenden Einflusses) und lässt sowohl die Bebußung des Tochterunternehmens nach dem Konzernumsatz ( 99 VerSanG-E) zu als auch eine Ausfallhaftung der Muttergesellschaft ( $\$ 7$ VerSanG-E). Die Einbeziehung des Konzernumsatzes entspricht der allgemeinen Rechtsentwicklung. So stellt etwa auch die Datenschutzgrundverordnung in Art. 83 auf den weltweiten Konzernumsatz ab. Dass dieses Abweichen von Grundsätzen des Konzernrechts, etwa vom Trennungsprinzip, im Hinblick auf das Schuldprinzip höchst problematisch ist, ${ }^{32}$ sei erwähnt, kann an dieser Stelle aber nicht vertieft werden. Die Problematik ist besonders virulent, wenn ohne das Erfordernis einer wirtschaftlichen Einheit die Bußgeldhöhe durch Umsätze anderer Rechtsträger bestimmt werden soll.

\subsection{Vorteilsbezogene Obergrenzen}

Noch unsystematischer und daher von besonderem Interesse ist die dritte mögliche Bestimmung der Obergrenze durch eine Multiplikation des erlangten Vorteils mit

28 Vgl. etwa TimmerbeIL, Sven - BlOME, Max-Niklas: Steter Tropfen höhlt den Stein - Die "wirtschaftliche Einheit" im deutschen Kartellrecht nach der 9. GWB-Novelle. BB, 2017, 1544, 1548 ff. m.w.N.

29 Vgl. insbes. EuGH, 10.09.2009, Rs. C-97/08-P, Slg. 2009 I-08237 Rn. 54 ff. (AKZO NOBEL NV u.a.); TIMMERBEIL - BLOME, BB, 2017, 1544, 1545.

30 Vgl. auch BuCK-HeEB, Petra - POELZIG, Dörte: Die Verhaltenspflichten ( $\S 63 \mathrm{ff}$. WpHG n. F.) nach dem 2. FiMaNoG - Inhalt und Durchsetzung. BKR, 2017, 485, 493; POELZIG, Dörte: Durchsetzung und Sanktionierung des neuen Marktmissbrauchsrechts. NZG, 2016, 492, 498.

31 BT-Drs. 19/23568.

32 TIMMERBEIL - BlOME, BB, 2017, 1544, 1548 f.; die rechtspolitische Sinnhaftigkeit bezweifeln BUCK-HEEB - POELZIG, BKR, 2017, 485, 493. 
einem bestimmten Faktor (sog. Multiplargeldbuße).$^{33}$ Der aus einem Verstoß erlangte Vorteil, der nach der üblichen Systematik für den abschöpfenden Teil einer Geldbuße maßgeblich ist, soll also im WpHG auch bestimmender Faktor für die Berechnung der Bußgeldrahmenobergrenze sein. ${ }^{34}$ Teilweise wird dies nicht als Obergrenze, sondern als Zumessungsgesichtspunkt betrachtet. ${ }^{35}$ Auch wenn diese Sicht einzelfallgerechtere Ergebnisse ermöglichen würde, bietet der Gesetzeswortlaut hierfür keinen Anhaltspunkt, sodass von drei alternativen Obergrenzen auszugehen ist. Während nach der herkömmlichen Struktur die Geldbuße den Vorteil abschöpfen und darüber hinaus einen ahndenden Teil enthalten soll (vgl. § 17 Abs. 4 OWiG), bestimmt sich hier die Geldbuße in ihrer Gesamtfunktion am Vorteil. ${ }^{36}$ Diese Obergrenze, die auch deutlich höher liegen kann als die alternativen Obergrenzen, wird ermittelt, indem der tatsächlich gezogene Vorteil mit einem vom Gesetz bestimmten Faktor multipliziert wird, und zwar entweder mit dem Faktor 1,5, 2 oder 3. Der wirtschaftliche Vorteil, der auch vermiedene Verluste umfasst, kann geschätzt werden, vgl. § $120 \mathrm{WpHG}$ Abs. 17-22a, jeweils S. 4. Diese Art der Höchstmaßbestimmung ist jedenfalls im Grundsatz europarechtlich begründet, etwa durch Art. 30 Abs. 2 lit. h der Marktmissbrauchsverordnung. ${ }^{37}$ Ähnliche Funktionen erfüllt auch die mehrerlösbezogene Obergrenze z. B. in $\S 172$ Abs. 3 Nr. 2 SAG seit 2014. Die vorteilsbezogene Obergrenze unterscheidet sich zwar stark von der Mehrerlösgrenze, entspricht ihr aber funktional und ist daher in ihrer dogmatischen Konzeption den gleichen Kritikpunkten ausgesetzt. ${ }^{38}$ Der Gedanke einer vorteils- oder erlösbezogenen Obergrenze ist aber nicht nur europarechtlich begründet. So sah bereits das Kartellrecht im GWB von 1958-2005 eine am Dreifachen des Mehrerlöses orientierte Obergrenze vor. ${ }^{39}$ Dieser Regelungsmechanismus wurde 2005 auch in $\S 95$ Abs. 2 EnWG übernommen. Auch international ist diese Bußgeldrahmenbestimmung durchaus

33 Systematisch unterscheiden sich Bruchteils- und Multiplargeldbußen nur insofern, als bei ersteren der Faktor unter 1 liegt. Daraus folgt aber praktisch, dass umsatz-, gewinn- und ertragsbasierte Geldbußen Bruchteilsgeldbußen sind, vorteils- und mehrerlösbasierte Geldbußen Multiplargeldbußen.

34 Vgl. etwa BaFin, WpHG-Bußgeldleitlinien II, Stand: Februar 2017, S. 6 f.

${ }^{35}$. Vgl. dazu SpOERR, a.a.O., WpHG § 120 Rn. 386, 379.

36 Vom zumindest primär strafenden Charakter spricht SPOERR, a.a.O., WpHG $\S 120$ Rn. 378.

37 VO (EG) Nr. 596/2014.

38 Unterschiede in Bezug auf den Bestimmtheitsgrundsatz sieht wegen der größeren Konturierung des Vorteilsbegriffs aber ACHENBACH, Hans: Verfassungswidrigkeit variabler Obergrenzen der Geldbußzumessung bei Kartellrechtsverstößen? WuW, 2002, 1154, 1160 f.; ders., wistra, 2018, 13, 19. Allerdings ist m.E. fraglich, ob die Argumentation zu $\S 17$ Abs. 4 OWiG wirklich übertragbar ist. Denn es ließe sich vertreten, dass der Bestimmtheitsgrundsatz beim abschöpfenden Teil einer Geldbuße anders auszulegen ist.

39 Vgl. zu diesbezüglichen Schwierigkeiten MEESSEN, Karl M.: Die 7. GWB-Novelle - verfassungsrechtlich gesehen. $W u W, 2004,733,739$ f.; zum Hintergrund der Abschaffung vgl. STAEBE, Erik: In: SÄCKER, Franz Jürgen (Hrsg.): Berliner Kommentar zum Energierecht. 4. Aufl., Frankfurt a. M., 2019, EnWG § 95, Rn. 40. 
verbreitet; etwa sieht der Insider Trading Sanctions Act der USA seit 1984 eine Orientierung am dreifachen Gewinn (profit) vor. ${ }^{40}$

Diese Art der Bußgeldrahmenbestimmung wirft, unabhängig davon, ob auf den Vorteil, den Mehrerlös oder den Gewinn, abgestellt wird, grundlegende Fragen auf.

Vielfach wird auch hier - ähnlich wie bei der umsatzorientierten Obergrenze die Einhaltung des Bestimmtheitsgrundsatzes bezweifelt. Weil die konkrete Obergrenze erst im Nachhinein bestimmt werden könne ${ }^{41}$ und zudem durch den Multiplikator ein erheblicher Spielraum für den Rechtsanwender bestehe, ${ }^{42}$ seien die verfassungsrechtlichen Grenzen insoweit nicht gewahrt. Der Spielraum, der durch den Multiplikator eröffnet wird, unterscheidet sich nicht prinzipiell von dem Spielraum, der stets bei der Strafzumessung oder der Bußgeldbemessung bestehen muss, um alle Faktoren des Einzelfalles berücksichtigen zu können. ${ }^{43}$ Die Tatsache, dass vor Tatbegehung keine absolute Obergrenze für die Bußgeldhöhe festgestellt werden kann, widerspricht aus den bereits genannten Gründen weder dem deutschen noch dem ohnehin weiteren europäischen Bestimmtheitsgrundsatz. ${ }^{44}$

Entscheidend scheint mir ein anderer Gesichtspunkt zu sein: Die Orientierung des abstrakten Bußgeldrahmens an dem konkret entstandenen Vorteil ist unsystematisch und dogmatisch nicht begründbar. Sie führt zu rein zufälligen Bußgeldrahmenverschiebungen. Der Gesetzgeber ist jedoch bei der Bestimmung eines Straf- oder Bußgeldrahmens gehalten, abstrakt die mögliche Schwere der Verstöße zu bestimmen und in der Rechtsfolge abzubilden. Es ist demgegenüber Aufgabe der Behörden und Gerichte bei der Bemessung die konkreten Umstände des Einzelfalles zu berücksichtigen. Es gibt aber keine Vermutung dafür, dass die abstrakte Schwere einer Ordnungswidrigkeit ein Mehrfaches des dabei konkret erlangten Vorteils wäre. ${ }^{45}$ Ein Vorteil kann ausbleiben, zufällig besonders groß ausfallen und ist schließlich eine rein einzelfallabhängige Größe. Er ist deswegen aus meiner Sicht als abstrakter Faktor des Bußgeldrahmens völlig ungeeignet, insbesondere zur Bestimmung einer abstrakten Obergrenze des ahndenden Teils einer Geldbuße.

Das gleiche Argument, dass also rein konkrete, später feststehende Faktoren zur Bestimmung eines abstrakten Bußgeldrahmens herangezogen werden, könnte man scheinbar auch bezüglich der Orientierung am Umsatz vorbringen. Auch diese Obergrenze ist von konkreten Umständen abhängig und steht erst später fest. Hier besteht

40 Sec. 2 of the Insider Trading Sanctions Act of 1984.

41 ACHENBACH, WM, 2018, 1337, 1340; anders wohl noch ders., wistra, 2018, 13, 19.

42 ACHENBACH, WM, 2018, 1337, 1340; ders., wistra, 2018, 13, 19; allg. zur Unbestimmtheit bei der Wahl eines Multiplikators auch ders., WuW, 2002, 1154, $1159 \mathrm{f}$.

43 BVerfGE 105, 35, 154 ff.; REMMERT, a.a.O., Art. 103 Abs. 2 Rn. 98 m.w.N.

44 Vgl. auch BÖSE - JANSEN, a.a.O., WpHG § 120 Rn. 96, die auch auf die fehlende Möglichkeit zur Verhängung von Freiheitsstrafen rekurrieren; vgl. auch BIERMANN, a.a.O., GWB § 81 Rn. 450.

45 Zum fehlenden Zusammenhang des Mehrerlöses und der Schuld auch MeEsSEN, $W u W$, 2004, 733, 742 . 
jedoch ein systematischer Unterschied, der eine Orientierung am Umsatz grundsätzlich zulässt; nur rechtstechnisch ist die Umsetzung bedenklich. Der Orientierung am Vorteil stehen demgegenüber grundsätzliche Bedenken entgegen.

Die umsatzorientierte Obergrenze dient einem im Kern nachvollziehbaren Gedanken: das Bußgeld soll bei gleich schwerwiegendem Verstoß gleich stark wirken, was von der wirtschaftlichen Leistungsfähigkeit des Verbands abhängt. ${ }^{46}$ Zwar hätten der europäische und deutsche Gesetzgeber dies handwerklich besser umsetzen können, etwa durch ein Tagessatzsystem wie bei der Geldstrafe oder wie auch im österreichischen Recht bei der Verbandsgeldbuße. ${ }^{47}$ Diese Systeme sehen zwar auch einen absolut bezifferten Tageshöchstsatz vor. ${ }^{48}$ Das ist aber rechtlich nicht geboten. ${ }^{49}$ Man dürfte meines Erachtens auch die Anzahl der Tagessätze im Höchstmaß absolut, die Höhe der Tagessätze aber rein relativ in Abhängigkeit vom Umsatz begrenzen, wie es ähnlich auch im nordrhein-westfälischen Entwurf für ein Verbandsstrafgesetzbuch vorgesehen war. ${ }^{50}$ Eine solche Lösung würde dem Bestimmtheitsgebot besser genügen als das derzeitige System. Es würde andererseits die Nachteile eines Systems mit absoluter Obergrenze vermeiden. Diese sieht etwa der Bundesgerichtshof darin, dass eine absolute Tagessatzobergrenze entweder so hoch angesiedelt sein müsste, dass sie unbestimmt wäre und reine Alibifunktion hätte oder andererseits so niedrig angesiedelt sein müsste, dass sie nicht zu gerechten Sanktionen umsatzstarker Verbände führen könnte. ${ }^{51}$ Zudem könnte man anstelle des Umsatzes Gewinn oder Ertrag heranziehen. Damit wäre man in der Konzeption aber, von Einzelheiten abgesehen, ${ }^{52}$ gar nicht weit entfernt von der bisherigen Lösung; es wäre nur eine andere, systematisch klarere, Regelungstechnik.

Es spricht jedoch kein abstrakter Strafzumessungs- bzw. Ahndungsgesichtspunkt für eine vorteilsbezogene Bestimmung der Bußgeldrahmenobergrenze. ${ }^{53}$ Der Vorteil als solcher ist zwar Bestandteil der Tatauswirkungen, nicht jedoch ein Vielfaches des Vorteils. Daher stellt sich die Frage, ob ein solcher Bußgeldrahmen das abstrakte Unrecht der Taten schuldangemessen abbildet. Das Schuldprinzip gilt auch im Ordnungswidrigkeitenrecht. ${ }^{54}$ Dabei limitiert es nicht nur die Bebußung im Einzelfall, sondern setzt auch Grenzen für die gesetzgeberische Bestimmung des Bußgeldrahmens in Form des Übermaßverbots, des Gebots des schuldangemessenen Strafens

46 Vgl. zu dieser Parallele zur Geldstrafe auch OLG Düsseldorf, 26. 06. 2009, Az.: 2a Kart $2-6 / 08$, juris, Rn. 610 im Vorgang zur Grauzementkartellentscheidung des BGH.

47 Vgl. § 4 VbVG-Österreich.

48 So etwa in $\S 4$ S. 2 VbVG-Österreich.

49 Vgl. aber HASSEMER, Winfried - DALLMEYER, Jens: Gesetzliche Orientierung im deutschen Recht der Kartellgeldbußen und das Grundgesetz. 2010, S. 42.

50 Vgl. § 6 VerbStrG-E, verfügbar unter https://www.landtag.nrw.de/portal/WWW/dokumentenarchiv/Dokument/MMI16-127.pdf (zuletzt aufgerufen am 30. 1. 2021).

51 Vgl. BGH NZKart 2013, 195, 199 - Grauzementkartell.

52 Insbes. von der Frage, ob der Konzernumsatz maßgeblich sein kann sowie ob Umsatz, Ertrag oder Gewinn als Maßstab interessengerechter sind.

53 Anders, jedenfalls beim Mehrerlös, wohl BIERMANN, a.a.O., GWB § 81 Rn. 449 f.

54 BVerfGE 9, 167, 169. 
bzw. Ahndens sowie des Gebots des sinn- und maßvollen Strafens bzw. Ahndens. ${ }^{55}$ Diese Bußgeldrahmenbestimmung würde das Schuldprinzip aber nur dann verletzen, wenn der vorgesehene Rahmen auch für die schwersten denkbaren Fälle nicht mehr tat- und schuldangemessen wäre, wobei der Gesetzgeber einen weiten Spielraum hat. Eine Verletzung des Schuldprinzips wird sich daher nur schwer darlegen lassen. Auch das Bundesverfassungsgericht geht (obiter) davon aus, dass es dem Gesetzgeber unbenommen sei, schon durch die gesetzliche Regelung das Bußgeld auf ein Mehrfaches des durch die Ordnungswidrigkeit erlangten Vorteils festzusetzen. ${ }^{56}$ Eher als die Frage der Verfassungsmäßigkeit bzw. der Vereinbarkeit mit europäischem Primärrecht im Hinblick auf das Schuldprinzip stellt sich die Frage regulatorischer Sinnhaftigkeit. Wegen der Zufälligkeit des erlangten Vorteils eignet er sich nicht zur abstrakten Bestimmung der Deliktsschwere. Die Wahl dieses Parameters und der Multiplikatoren erinnert daher stark an ältere Rechtsordnungen, bei denen die Schuld oder auch individuelle Umstände eine geringere Rolle spielten. So sah insbesondere das römische Recht in der (privatrechtlichen) actio furti vor, dass der Dieb, abhängig von weiteren Umständen, den zwei- bis vierfachen Wert der Sache zu ersetzen habe. ${ }^{57}$ Auch dem deutschen Strafrecht waren Multiplarstrafen nicht unbekannt, etwa im Steuerstrafrecht der Reichsabgabenordnung ${ }^{58}$ oder im Zollstrafrecht (im allgemeinen Teil seit 1921 zunächst außerhalb des RStGB, ${ }^{59}$ dann in $§ 27$ Abs. $2,{ }^{60}$ später Abs. 3 RStGB) ${ }^{61}$ Sie wurden aber nach und nach, ${ }^{62}$ später auch wegen Bedenken im Hinblick auf das Schuldprinzip ${ }^{63}$ abgeschafft. Durch die Multiplikation des Vorteils fehlt nicht nur der Bezug zum Unrecht; das Ergebnis ist zudem

55 Vgl. hierzu BVerfG NJW, 1990, 1900, 1901, wo auf die zu Strafen entwickelte Rspr., etwa BVerfG $N J W, 1970,1453$, Bezug genommen wird; BVerfG $N J W, 1987,43,48$; NJW, 1990 31, 38; NJW, 1992, 2947, 2948; BVerfGE 105, 35.

56 Vgl. BVerfG NJW, 1990, 1900, 1901.

57 Vgl. KASER, Max: Das römische Privatrecht-Erster Abschnitt. 2. Aufl., 1971, S. 616.

58 Z. B. § 373 AO i.d.F. v. 1919. Zur früheren Rechtsentwicklung HIS, Rudolf: Das Strafrecht des deutschen Mittelalters, Zweiter Teil: Die einzelnen Verbrechen. 1935, 57 ff.; vgl. auch RÜPING, Hinrich: Geldstrafe und Buße. Zur Entwicklung der öffentlichen Strafe. ZStW, 85 (1973), 672, $684 \mathrm{f}$.

59 Vgl. $§ 1$ Abs. 2 des Gesetzes zur Erweiterung des Anwendungsgebiets der Geldstrafe und zur Einschränkung der kurzen Freiheitsstrafen, RGB1. I, 1921, 1604; vgl. hierzu FRANK, Reinhard: Das Strafgesetzbuch für das Deutsche Reich. 18. Aufl., Tübingen, 1931, § 27 I, $\S 1$ I 3 .

60 Vgl. RGBl. I, 1923, S. 254.

61 Vgl. RGBl. I, 1923, S. 1118; 1924, S. 44.

62 Vgl. zur Abschaffung in der Reichsabgabenordnung RBGl. I, 1939, S. 1181; insgesamt seit 1974 durch Art. 12 Abs. 2, 4 EGStGB.

63 Vgl. NIESE, ZStW 70 (1958), 337 (338); RUTKOWSKI - GÖHLER - BUDDENDIEK - LENZEN, Multiplarstrafen. In: BUDDENDIEK - RUTKOWSKI: Lexikon des Nebenstrafrechts. 42. EL 2019; vgl. auch BT-Drs. III/2150, S. 163: „,mit dem Grundgedanken der Geldstrafenneuregelung [...] unvereinbar“. Demgegenüber sieht KLUSMANN, Martin: In: WIEDEMANN, Gerhard (Hrsg.): Handbuch des Kartellrechts. 4. Aufl., München, 2020, § 57 Rn. 93, wohl nur fixe Multiplarstrafen als problematisch an. 
auch von der wirtschaftlichen Leistungsfähigkeit der Adressaten vollständig abgekoppelt und trifft diese daher gänzlich unterschiedlich.

Das moderne Recht ermöglicht es jedoch, zwischen Abschöpfungs- und Ahndungsfunktion zu unterscheiden. Während der Vorteil zur abstrakten Bestimmung der Abschöpfung naturgemäß geeignet ist (und diese die Adressaten auch gleich trifft, indem sie verhindert, dass Verstöße sich wirtschaftlich lohnen), sollte der Vorteil bei der Ahndungsfunktion nur als ein Element bei der konkreten Bußgeldzumessung herangezogen werden. Die notwendige Unterscheidung zwischen der Abschöpfungs- und der Ahndungsfunktion wird sonst grundlos aufgegeben. Dadurch werden die im Ordnungswidrigkeitenrecht ohnehin bestehenden Schwierigkeiten noch verstärkt. Nach den allgemeinen Regeln ist die Abschöpfung durch die Geldbuße die Regel, eine Einziehung nach $\S \S 29 a, 30$ OWiG subsidiär und damit die Ausnahme. Bei gewöhnlichen Geldbußen ist jedoch immerhin durch die Begründung klarzustellen, welcher Teil der Geldbuße abschöpfend ist und welcher ahndend. Dies gilt nach vorzugswürdiger Ansicht nicht nur für die gerichtliche Entscheidung, ${ }^{64}$ sondern bereits für den Bußgeldbescheid..$^{65}$ Dabei soll nach $\S 17 \mathrm{Abs.} 4 \mathrm{OWiG}$ der wirtschaftliche Vorteil im Wege einer Zumessungsrichtlinie die Untergrenze bilden, auch wenn in der Praxis dennoch vielfach auf einen abschöpfenden Teil verzichtet wird. ${ }^{66}$ Die im WpHG erfolgende Vermengung von abschöpfendem und ahndendem Teil ${ }^{67}$ einer Geldbuße ist jedoch besonders problematisch. So ist bereits unklar, ob $\S 17$ Abs. 4 S. 1 OWiG (ggf. i.V.m. $§ 30$ Abs. 3 OWiG) eingreift, also die Geldbuße regelmäßig über dem erlangten Vorteil zu liegen hat, oder ob demgegenüber eine rein ahndende Geldbuße verhängt werden darf, die darunter liegt. Im SAG und im KWG hat der Gesetzgeber zusätzlich zur Mehrerlösgrenze eine dem $\S 17$ Abs. 4 S. $1 \mathrm{OWiG}$ entsprechende Vorschrift vorgesehen; zudem wird dort angeordnet, § 17 Abs. 4 OWiG bleibe unberührt (vgl. $\S \S 172$ Abs. 3 SAG, 56 Abs. 7 KWG) ${ }^{68}$ Eine vergleichbare Regelung gibt es in $\S 120 \mathrm{WpHG}$ nicht; auch ist der Vorteil insgesamt für die Bußgeldrahmenobergrenze einschlägig. Einiges spricht daher dafür, nicht von einer solchen Untergrenze, sondern von einer abschließenden Regelung, auszugehen. ${ }^{69}$ Insbesondere würde auch die Kombination von $\S 17$ Abs. 4 S. 2 OWiG (Überschreitung des Höchstmaßes zur Vorteilsabschöpfung) mit einer vorteilsbezogenen Obergrenze von vornherein gegenstandslos sein. In Bereichen, in welchen

64 So BGH NStZ 2006, 231, 232 f., allerdings vorwiegend aus steuerrechtlichen Gründen; auf diese Gründe beschränkt BGH, Beschl. v. 17. 1. 2013, Az. 3 StR 167/13, BeckRS 2014, 5757, Rn. 43; vgl. auch BVerfG NJW 1990, 1900, 1901.

65 Jedenfalls bei erheblichen Vorteilen: BeckOK OWiG/SACKREUTHER, § 17 Rn. 134 m.w.N.; insoweit ist $\S 66$ Abs. $3 \mathrm{OWiG}$ aus rechtsstaatlichen Gründen einschränkend auszulegen.

66 Vgl. etwa für das Kartellrecht BACH: In: FS Canenbley. 2012, 15, 20.

67 Vgl. auch ACHENBACH, WM, 2018, 1337, 1340.

68 Zu Schwierigkeiten der Auslegung bei diesen Vorschriften Achenbach. wistra, 2018, 13, $20 \mathrm{f}$.

69 Vgl. SPOERR, a.a.O., WpHG $§ 120$ Rn. 386; unklar insoweit BaFin, WpHG-Bußgeldleitlinien II, Stand: Februar 2017, S. 9; a.A.: BÖSE - JANSEN, a.a.O., WpHG § 120 Rn. 101. 
mehrerlösbezogene Obergrenzen bestanden, wird § 17 Abs. 4 OWiG teilweise ohne besondere Anordnung für anwendbar gehalten $;{ }^{70}$ allerdings besteht ein Unterschied zwischen Mehrerlös und Vorteil, der einen Unterschied rechtfertigen kann. ${ }^{71}$ Zudem schreibt auch die Marktmissbrauchsverordnung keine solche Untergrenze vor; nach Art. 30 Abs. 2 lit. b muss nur die Möglichkeit der Einziehung bestehen, nach lit. h die Möglichkeit einer vorteilsbezogenen Obergrenze.

Unklar ist bei der Bebußung nach den drei alternativen Obergrenzen aber auch das Verhältnis zu etwaigen Schadensersatzleistungen. Jedenfalls, soweit dieser bereits geleistet oder unanfechtbar festgesetzt wurde ${ }^{72}$ ist dies bei der Bestimmung des Vorteils im Rahmen von $\S 17$ Abs. 4 OWiG zu berücksichtigen und kann sich erheblich auswirken. Konsequent müsste sich bei der vorteilsbezogenen Obergrenze im WpHG der Bußgeldrahmen nach unten verschieben, obschon es sich um einen abstrakten, insgesamt der Ahndung dienenden Bußgeldrahmen handelt. Ähnliche Probleme ergeben sich, wenn Schadensersatz erst nach der Bußgeldentscheidung geleistet wird. Man könnte auch hier - wie im Rahmen von $\S 17$ Abs. 4 OWiG $^{73}-\S 99$ Abs. 2 OWiG analog anwenden mit der Folge, dass dieser Betrag vom abschöpfenden Teil abgezogen bzw. rückerstattet würde. Materiell ist dies überzeugend. Insbesondere solange noch kein Schadensersatz geleistet wurde, könnte ansonsten sogar eine wirtschaftliche Gefährdung durch die Geldbuße eintreten, die Schadensersatzzahlungen erschwert. Konstruktiv ist aber auch dies wegen der Ahndungsfunktion der vorteilsbezogenen Bußgeldrahmenbestimmung im $\mathrm{WpHG}$ wiederum schwer zu begründen. ${ }^{74}$ All diese Erwägungen setzen aber voraus, dass Schadensersatzansprüche überhaupt bestehen, etwa nach $\S 826 \mathrm{BGB}$, nach $\S \S 97,98 \mathrm{WpHG}$ bei ad-hoc-Publizitäts-Pflichtverstößen, oder dass Schutzgesetze nach $\S 823$ Abs. 2 BGB verletzt wurden, was etwa bei der Marktmanipulation überwiegend nicht angenommen wird. ${ }^{75}$ Daher mögen die praktischen Konsequenzen begrenzt sein; diese Problematik besteht jedoch ganz generell bei der gewählten Regelungstechnik. Insofern ist am Entwurf des Verbandssanktionengesetzes zumindest positiv zu bewerten, dass es den abschöpfenden Anteil konsequent aufgibt und regelmäßig eine Abschöpfung neben der Sanktion fordert. ${ }^{76}$

70 So für $\S 81$ GWB i.d.F. bis 2005, vgl. BACH: In: FS Canenbley. 2012, 15, 19; die Rspr. vertrat auch den Standpunkt, es müsse mindestens in Höhe des Mehrerlöses bebußt werden, vgl. BGH NJW 3792, 3795 - Papiergroßhandel.

71 Vgl. auch BMJV, Handbuch des Nebenstrafrechts. 3. Aufl., 2018, Rn. 236 dazu, dass der Vorteil den dreifachen Mehrerlös übersteigen könne.

72 Vgl. MitsCH, a.a.O., § 17 Rn. 129 m.w.N.

73 Vgl. dazu MiTsCH, a.a.O., § 17 Rn. 129 m.w.N.; BACH: In: FS Canenbley. 2012, 15,19 f.

74 Für $\S 81$ GWB i.d.F. bis 2005 hat der BGH Schadensersatz aber als Argument für eine reine Ahndungsgeldbuße gelten lassen, vgl. BGH NJW 3792, 3795 - Papiergroßhandel.

75 Vgl. etwa WoRms, Alexander, in: Assmann, Heinz Dieter - SCHÜTZE, Rolf A. - BuCKHEEB, Petra (Hrsg.): Handbuch des Kapitalanlagerechts. 5. Aufl., München, 2020, § 10 Rn. 106 m.w.N.; vgl. allgemein ReUTER, Alexander: Schadensersatz und Bußgelder zu Lasten des Unternehmens bei Ad hoc-Pflichtverstößen: Ein Verstoß gegen die Grundrechte und die Treuepflicht der Aktionäre? NZG, 2019, 321.

76 Vgl. BT-Drs. 19/23568 S. 51, 68, 120. 


\section{FAZIT}

Zusammenfassend lässt sich sagen, dass der Bußgeldrahmen bei Sanktionen im Finanzmarkt mehrere problematische Tendenzen aus der Rechtsentwicklung der letzten Zeit miteinander vereint. Die alternative Höchstmaßbestimmung, die an unsystematische Faktoren anknüpft, lässt den Eindruck entstehen, ein möglichst hoher Bußgeldrahmen sei in jedem Fall und unabhängig von sachlichen oder systematischen Argumenten positiv zu beurteilen. Gerade die gesetzgeberische Entscheidung, dass zwingend der höchste der drei Werte für die Behörde bzw. den Richter als Bußgeldrahmenobergrenze maßgeblich sein soll, überzeugt nicht. Diese Wertung ist auch nur zu einem geringen Teil europarechtlich determiniert; zum überwiegenden Teil hätte der deutsche Gesetzgeber - auch in europarechtskonformer Weise ${ }^{77}$ - einen der Werte wählen oder die Wahl des Rahmens in das Ermessen des Tatrichters ${ }^{78}$ stellen können.

Dies ist insbesondere deshalb erstaunlich, weil viele der bereits unabhängig davon zur Verfügung stehenden Mechanismen praktisch nicht genutzt werden, sodass an der Notwendigkeit Zweifel angebracht sind. Insbesondere die Renaissance der überwunden geglaubten Multiplargeldbußen, die neuerdings auf europäischer Ebene vorangetrieben wird, ist vor diesem Hintergrund kritisch zu sehen. Vorzugswürdig erscheint ein tagessatzbasierter Bußgeldrahmen sowie eine klare Differenzierung zwischen Ahndung und Abschöpfung.

\section{LITERATURVERZEICHNIS}

[1] ACHENBACH, Hans: Das Höchstmaß der Verbandsgeldbuße wegen Kapitalmarkt-Straftaten. WM, 2018, $1337 \mathrm{ff}$.

[2] ACHENBACH, Hans: Neue Sanktionen im Finanzmarktrecht - alte und neue Zweifelsfragen. wistra, 2018, $13 \mathrm{ff}$.

[3] ACHENBACH, Hans: Verfassungswidrigkeit variabler Obergrenzen der Geldbußzumessung bei Kartellrechtsverstößen? WuW, 2002, 1154 ff.

77 So bzgl. der umsatzbezogenen und absoluten Obergrenze TEIGELLACK, Lars - DolfF, Christian: Kapitalmarktrechtliche Sanktionen nach dem Regierungsentwurf eines Ersten Finanzmarktnovellierungsgesetzes - 1. FiMaNoG. $B B, 2016,387,390$ unter Verweis darauf, dass die VO (EG) Nr. 596/2014 - anders als die Transparenzrichtlinie - die Maßgeblichkeit des höheren Wertes nicht vorschreibt; Stellungnahme des DAI zum Referentenentwurf zum Finanzmarktnovellierungsgesetz vom 18. 11. 2015, S. 14, unter https:// www.dai.de/files/dai_usercontent/dokumente/positionspapiere/2015-11-18\%20DAIStellungnahme\%20FimanoG.pdf (zuletzt aufgerufen am 30.01.2021); auch bezüglich der vorteilsbezogenen Obergrenze in Art. 30 Abs. 2 lit. ha VO (EG) Nr. 596/2014 gibt es nur die Verpflichtung, diese Möglichkeit der Behörde einzuräumen; die Maßgeblichkeit des höheren Betrags ist dort nicht vorgesehen.

$78 \mathrm{Zu}$ diesem Gedanken SPOERR, a.a.O., WpHG § $120 \mathrm{Rn}$. 383, der dies allerdings als eine Frage des Regulierungsstils einordnet. 
[4] Assmann, Heinz Dieter - SchneIder, Uwe H. - MÜlbert, Peter O. (Hrsg.): Wertpapierhandelsrecht. 7. Aufl., Köln, 2019.

[5] Assmann, Heinz Dieter - SchÜtze, Rolf A. - Buck-HeEB, Petra (Hrsg.): Handbuch des Kapitalanlagerechts. 5. Aufl., München, 2020.

[6] BACH, Albrecht: Kartellbußgelder und Schadensersatz: Ansätze zur Konfliktlösung. In: Studienvereinigung Kartellrecht e.V. (Hrsg.): Festschrift für Canenbley. München, 2012, 15 ff.

[7] BARTH, Christoph - BUDDE, Stephanie: „Die Strafe soll nicht größer sein als die Schuld“ Zum Urteil des BGH in Sachen Grauzement und den neuen Leitlinien für die Bußgeldzumessung. NZKart, 2013, $311 \mathrm{ff}$.

[8] Buck-Heeb, Petra - Poelzig, Dörte: Die Verhaltenspflichten (§§ $63 \mathrm{ff}$. WpHG n. F.) nach dem 2. FiMaNoG - Inhalt und Durchsetzung. BKR, 2017, $485 \mathrm{ff}$.

[9] BUDDENDIEK, Hans - RUTKOWSKI, Jörg (Hrsg.): Lexikon des Nebenstrafrechts. München, 42, EL 2019.

[10] BÜLTE, Jens: Ordnungswidrigkeitenrecht. 6. Aufl., München, 2020.

[11] Bundesanstalt für Finanzdienstleistungsaufsicht: WpHG-Bußgeldleitlinien II. Stand: Februar 2017.

[12] Bundesministerium der Justiz und für Verbraucherschutz: Handbuch des Nebenstrafrechts. 3. Aufl., 2018.

[13] CIRENER, Gabriele - RADTKE, Henning - RISSING-VAN SAAN, Ruth RÖNNAU, Thomas - SCHLUCKEBIER, Wilhelm (Hrsg.): Strafgesetzbuch Leipziger Kommentar. Großkommentar. Bd. 1, 13. Aufl., Berlin, 2020.

[14] DANNECKER, Gerhard: Nullum crimen, nulla poena sine lege und seine Geltung im Allgemeinen Teil des Strafrechts. In: DANNECKER, Gerhard (Hrsg.): Festschrift für Otto. Köln, 2007, 25 ff.

[15] Deutsches Anwaltsinstitut: Stellungnahme zum Referentenentwurf zum Finanzmarktnovellierungsgesetz vom 18. 11. 2015, unter https://www.dai.de/files/dai_usercontent/dokumente/positionspapiere/2015-11-18\%20DAI-Stellungnahme\%20FimanoG.pdf (zuletzt aufgerufen am 30. 1. 2021).

[16] FRANK, Reinhard: Das Strafgesetzbuch für das Deutsche Reich. 18. Aufl., Tübingen, 1931.

[17] GRAF, Jürgen Peter (Hrsg.): BeckOK OWiG. München, 2021.

[18] HASSEMER, Winfried - DALLMEYER, Jens: Gesetzliche Orientierung im deutschen Recht der Kartellgeldbußen und das Grundgesetz. Baden-Baden, 2010.

[19] HIs, Rudolf: Das Strafrecht des deutschen Mittelalters. Zweiter Teil: Die einzelnen Verbrechen, Weimar, 1935. 
[20] IMMENGA, Ulrich - MESTMÄCKER, Ernst-Joachim (Hrsg.): Wettbewerbsrecht. 6. Aufl., München, 2020.

[21] Kallmayer, Axel - Haupt, Heiko: Die Urteile des EuG zum Fernwärmerohrkartell - Die Bußgeldleitlinien der Kommission auf dem Prüfstand. EuZW, 2002, $677 \mathrm{ff}$.

[22] KASER, Max: Das römische Privatrecht - Erster Abschnitt. 2. Aufl., München, 1971.

[23] MAUNZ, Theodor-DÜRIG, Günter (Begr.): Grundgesetz. Loseblatt, Stand: 92. EL 2020.

[24] MEESSEN, Karl M.: Die 7. GWB-Novelle - verfassungsrechtlich gesehen. $W u W, 2004,733 \mathrm{ff}$.

[25] MEYER, Andreas - VEIL, Thomas - RÖNNAU, Rüdiger (Hrsg.): Handbuch zum Marktmissbrauchsrecht. München, 2018.

[26] MITSCH, Wolfgang (Hrsg.): Karlsruher Kommentar zum Gesetz über Ordnungswidrigkeiten. 5. Aufl., München, 2018.

[27] NIESE, Wemer: Das Steuerstrafverfahren. ZStW, 70 (1958), 337 ff.

[28] PoElZIG, Dörte: Durchsetzung und Sanktionierung des neuen Marktmissbrauchsrechts. NZG, 2016, $492 \mathrm{ff}$.

[29] REUTER, Alexander: Schadensersatz und Bußgelder zu Lasten des Unternehmens bei Ad hoc-Pflichtverstößen: Ein Verstoß gegen die Grundrechte und die Treuepflicht der Aktionäre? NZG, 2019, $321 \mathrm{ff}$.

[30] RÜPING, Hinrich: Geldstrafe und Buße. Zur Entwicklung der öffentlichen Strafe. ZStW, 85 (1973), 672 ff.

[31] SÄCKER, Franz Jürgen (Hrsg.): Berliner Kommentar zum Energierecht. 4. Aufl., Frankfurt a. M., 2019.

[32] SATZGER, Helmut: Mutual Recognition in Times of Crisis - Mutual Recognition in Crisis? An Analysis of the New Jurisprudence on the European Arrest Warrant. EuCLR, 2018, 317 ff.

[33] SCHWARK, Eberhard - ZIMMER, Daniel (Hrsg.): Kapitalmarktrechtskommentar. 5. Aufl., München, 2020.

[34] Staub, Hermann (Begr.): Handelsgesetzbuch. Bd. 7/2, 5. Aufl., Berlin, 2012.

[35] Szesny, André M. - KUTHE, Thorsten (Hrsg.): Kapitalmarkt Compliance. Heidelberg, 2. Aufl., 2018.

[36] Teigellack, Lars - DolfF, Christian: Kapitalmarktrechtliche Sanktionen nach dem Regierungsentwurf eines Ersten Finanzmarktnovellierungsgesetzes - 1. FiMaNoG. BB, 2016, $387 \mathrm{ff}$. 
[37] TimmerbeIL, Sven - Blome, Max-Niklas: Steter Tropfen höhlt den Stein Die "wirtschaftliche Einheit" im deutschen Kartellrecht nach der 9. GWBNovelle, BB 2017, S. 1544.

[38] VOET VAN VoRMIZEELE, Philipp: Kartellrecht und Verfassungsrecht, NZKart 2013, $386 \mathrm{ff}$.

[39] MonTAG, Frank - SÄCKER, Franz Jürgen - BIEN, Florian - MeIER-BeCK, Peter (Hrsg.): Münchener Kommentar zum Wettbewerbsrecht, 3. Aufl., München 2020 .

[40] Wiedemann, Gerhard (Hrsg.): Handbuch des Kartellrechts, 4. Aufl., München 2020. 